\title{
Effect of a therapeutic dose of pseudoephedrine on swimmers' performance
}

P J-L Gradidge, ${ }^{1}$ MSc (Med) (Biokinetics); D Constantinou, ${ }^{1}$ MB BCh, BSc Med (Hons), FFIMS; S-M Heard, ${ }^{1}$ BHSc (Hons) (Biokinetics); C King, ${ }^{1}$ BHSc (Hons) (Biokinetics); H Morris-Eyton, ${ }^{2}$ MEd (Adult Education)

${ }^{1}$ Centre for Exercise Science and Sports Medicine, School of Therapeutic Sciences, Faculty of Health Sciences, University of the Witwatersrand,

Johannesburg, South Africa

${ }^{2}$ School of Education, Faculty of Humanities, University of the Witwatersrand, Johannesburg, South Africa

Corresponding author: P J-L Gradidge (philippe.gradidge@wits.ac.za)

Background. The potential performance-enhancement effect of pseudoephedrine (PSE) use has led to its prohibition in competition sports (urine concentrations $>150 \mu \mathrm{g} / \mathrm{ml}$ ). Data are, however, scarce regarding whether therapeutic PSE use enhances swimming performance. Objective. To investigate the effect of therapeutic PSE use on performance in aerobic and explosive sprint swimming events.

Method. A double-blinded cross-over study design was used. Participants in the control group initially received a placebo and those in the experimental group received a divided PSE dose of $90 \mathrm{mg} / \mathrm{d}$. Anaerobic power (50 m sprint) and aerobic (2 $000 \mathrm{~m})$ swimming testing was conducted at (i) baseline; (ii) after ingestion of a placebo or PSE; and (iii) after the groups were crossed over, following a wash-out period of 4 days, to determine changes in performance between trials.

Results. The participants (mean age 44 years; $N=7$ ) were competitive masters swimmers with normal resting heart rates ( 68 beats per minute (bpm); standard deviation (SD) \pm 14 ) and blood pressures (BPs) (171 (SD \pm 27$) / 83(\mathrm{SD} \pm 16) \mathrm{mmHg})$. The use of PSE during the anaerobic swim test showed only a trivial chance (68\%) of improvement, with a likely enhancement in systolic BP (86\%). The aerobic swim test did not affirm performance enhancement as measured by time to completion ( $52 \%$ chance of a positive effect; $41 \%$ chance of a negative effect), nor did any other physiological variable of interest (peak heart rate and exercising BP) differ significantly from baseline results.

Conclusion. The use of a therapeutic amount of PSE in short and endurance swimming trials did not appear to have any major ergogenic effect on performance.

S Afr J SM 2013;25(2):43-46. DOI:10.7196/SAJSM.378 


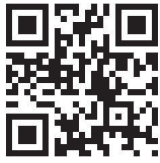

Pseudoephedrine (PSE) is a sympathomimetic substance found in over-the-counter (OTC) products such as nasal decongestants, and in respiratory medicines in combination with antihistamines. ${ }^{[1]}$ The action of sympathomimetic substances mimics that of epinephrine and norepinephrine, acting mainly on the $\alpha$ - and $\beta$-adrenoreceptors. ${ }^{[2]}$ The ingestion of PSE results in the release of norepinephrine from storage sites in nerve and neural tissue. ${ }^{[2]}$ This is thought to result from direct stimulation of post-synaptic receptors and inhibition of neurotransmittor reuptake ${ }^{[3]}$ It is also hypothesised that PSE has iono- and chronotropic effects on the heart and that it increases the exercising heart rate (HR), resulting in greater venous return and cardiac output. ${ }^{[2,3]}$ The latter is thought ultimately to result in increased oxygenated blood supply to the exercising muscles, reducing the premature onset of muscle fatigue. The total torque production of the muscles is consequently increased and may result in enhanced athletic performance. ${ }^{[2,3]}$ These and other positive effects of PSE appeal to athletes, although doses $\geq 240 \mathrm{mg}$ (above therapeutic doses) may be needed for a positive ergogenic effect. ${ }^{[4,5]}$

Literature on the effectiveness of PSE as a performance enhancer is conflicting. Some studies have shown that PSE use improves $1500 \mathrm{~m}$ running times, maximal torque in isometric knee extension, peak power during maximal cycling performance and lung-function capacity. ${ }^{[2,6]} \mathrm{On}$ the contrary, other studies have shown that therapeutic doses of PSE have no effect on cycling performance, $5000 \mathrm{~m}$ endurance running, handgrip maximal voluntary contraction, time to fatigue, and peak mean total power output during anaerobic cycling. ${ }^{[1,3,5]}$ The World AntiDoping Agency (WADA) decided to prohibit PSE use in sport because of evidence that it does, or has the potential to enhance performance; ${ }^{[7,8]}$ however, confirmation of the ergogenic effect is limited and therapeutic use of PSE as a potential performance enhancer has only been examined in a small number of studies. Furthermore, research on its use in swimmers is limited. The purpose of this study was therefore to investigate the effect of the therapeutic use of PSE on swimming performance in aerobic and anaerobic (explosive sprint) swimming events.

\section{Methods}

We used a double-blinded, randomised, controlled cross-over trial design of repeated measures. Ethical approval of the study was granted by the University of the Witwatersrand (M1100445).

\section{Participants}

Male and female masters-level swimmers competing in swimming events were invited to participate in the study $(N=7)$. Inclusion criteria included an appropriate level of swimming, age 25 - 60 years (as defined by the Federation Internationale De Natation (FINA) ${ }^{[9]}$ ), a training frequency of $\geq 3$ days per week, and a minimum of 2 years of swimming experience in masters-level competitive swimming events. Exclusion criteria included a history of or current cardiac disease, congenital defects, hypertension or renal disease; recent illness; the presence of musculoskeletal injury; and the existing use of performance-enhancing substances or recreational drugs.

Participants were initially randomly assigned to an experimental group (PSE) or a control group (placebo). They were then asked to perform anaerobic power (50 m sprint) and aerobic $(2000 \mathrm{~m}) \mathrm{swim}$ tests at $(i)$ baseline; (ii) after ingestion of the placebo or PSE; and (iii) after the groups were crossed over, following a wash-out period of 4 days, to determine changes in performance between trials.

To prevent a trained performance increase, the swimmers were asked to maintain their training regimens during the testing period. Their diets remained the same, except that they were asked to avoid caffeine, alcohol, nicotine and other stimulant drugs for 24 hours prior to testing.

\section{Anthropometrical measurements}

Resting and post-exercise HRs were measured using the radial pulse after a 5 - 10-minute resting period with participants seated. ${ }^{[10]}$ Resting blood pressure (BP) was then measured using an aneroid sphygmomanometer and accompanying stethoscope (Delux (KT-102) Rappaport, Hi-care). BP was recorded immediately after the aerobic and anaerobic swim tests. ${ }^{[10]}$ Height was measured using a stadiometer. ${ }^{[10]}$ Weight was measured to the nearest $\mathrm{kg}$ with participants dressed in minimal clothing and with shoes removed (Seca, Germany).

Following baseline measurements, the participants were randomly assigned to the control and experimental groups. After a warm-up period to which they were individually accustomed, the participants were asked to perform the baseline aerobic and anaerobic swimming tests. The post-exercise HR, BP, rate of perceived exertion (RPE) (using the Borg 1-10 RPE scale $)^{[10]}$ and time taken to perform the tests (Econosport 240 stopwatch, Sportline) were recorded by the same researcher.

\section{Swimming performance tests}

Although the participants were familiar with the test protocols, and therefore knowledgeable regarding procedural expectations, the protocols were fully explained before each testing session to confirm full comprehension of participant requirements. Baseline testing served to acquaint the participants with the tests, and the results thereof allowed for comparison with the subsequent swim tests.

The first test involved an anaerobic power activity, with participants required to give maximal effort in a $50 \mathrm{~m}$ sprint (2 lengths of a standard $25 \mathrm{~m}$ pool). The second swim test comprised a timed $2 \mathrm{~km}$ aerobic swim, again required to be performed with maximal effort. ${ }^{[5]}$ In the latter, the best time taken for the swimmer to complete the test was recorded, and the researchers counted the number of laps performed ${ }^{[1]}$

The tests were conducted at different swimming pools as participants belonged to different swim clubs; however, the aerobic and anaerobic tests were conducted in the same swimming pool per participant. Likewise, the tests were conducted at the same time of day per participant. To keep procedures consistent, the swimmers were required to start tests in the swimming pool and push off the pool wall. $\mathrm{HR}$ and BP were recorded before and immediately after each test. No verbal encouragement was given to participants to ensure consistency in this regard throughout testing. Participants were not given individual results, and were reminded that the data were analysed per group.

\section{Placebo and PSE administration}

After baseline data collection, each swimmer received either the placebo (no active ingredient) or $30 \mathrm{mg}$ of PSE (Sudafed, Pfizer) 3 times daily for 4 days (to ensure a therapeutic dose). The researchers and participants were blinded to which drug each group received. A sufficient supply of tablets for 4 days was administered to each participant by a research assistant. The placebos and active drugs were coded by independent persons, allowing for decoding at the end of the study. 
To improve compliance, the participants received a daily text message via mobile phone, reminding them to ingest the tablet at the correct time of day. After taking the treatment for 4 days, the swimmers were tested in the same manner as for baseline testing. A wash-out period of 4 days was then observed to allow for complete elimination of the substances. The participants were subsequently crossed over, and the same procedures followed after ingestion of the opposite treatment (i.e. placebo or PSE).

\section{Statistical analysis}

Descriptive statistics were used for demographic data. Non-parametric statistics were used, as data were not normally distributed and the sample size was small $(N=7)$. The Friedman analysis of variance (ANOVA) with Kendall co-efficient of concordance was used to measure the association between paired samples using Statistica (version 10). Paired $t$-tests were done to determine the effect statistic and $p$-value for each variable of interest. The magnitude of difference was calculated accordingly to determine if changes were 'positive', 'trivial' or 'negative' according to Batterham and Hopkins ${ }^{[11]}$ (a probability was ascribed to each magnitude). Cohen's $d$ was used to determine effect size between placebo and PSE use.

\section{Results}

Seven masters-level competitive swimmers participated in the investigation. Participant demographics and baseline data are displayed in Table 1. The mean age (44 years; standard deviation (SD) \pm 7 ) represented a typical team at the masters stage of a swimming career. The swim times in the $50 \mathrm{~m}$ and $2000 \mathrm{~m}$ protocols did not change in a meaningful way, reflected by the small effect sizes $(d=0.18-0.06)$ and the magnitude inferences (\%positive/trivial/negative effects) of $\% 52 / 8 / 41$ and $\% 25 / 68 / 8$, respectively (Table 2 ). HR did not change significantly in the $50 \mathrm{~m}$ time trial, but was likely to have increased in the $2000 \mathrm{~m}$ swim (\%85/6/9). This is supported by the small to moderate effect size shown in both tests $(d=0.3-0.5)$. The majority of the other findings had effect sizes that were small; however, diastolic blood pressure (DBP) had medium effect sizes in the anaerobic and aerobic swim tests ( $d=0.76$ and 0.56 , respectively). The RPE during all trials was near maximal effort, as was requested of the participants, and did not change significantly throughout (small effect size; $d=0.2$ ). Finally, there may have been a likely change in systolic blood pressure (SBP) in the $50 \mathrm{~m}$ sprint (\%86/11/3) and $2000 \mathrm{~m}$ time trial (\%76/17/7).

\section{Discussion}

Athletes are continually looking for ways to enhance their performance in order to gain a competitive advantage in sport; and anti-doping organisations such as WADA are continually trying to keep abreast of these means. ${ }^{[12]}$ Some athletes use well-known OTC medicines that have been prohibited as they have been shown to enhance performance. ${ }^{[3,12]}$ Others, to aid with recovery and training, consume nutritional supplements that are potentially contaminated, knowingly or inadvertently, with prohibited substances. ${ }^{[13]}$ In both cases, the athlete could be sanctioned if doping tests are positive; the former scenario being of particular concern, as PSE can be found in nasal decongestants and respiratory medicines in combination with antihistamines. ${ }^{[1]} \mathrm{A}$ recently published position statement argued that although OTC substances such as PSE could potentially augment performance, these products could cause long-lasting harm to athletes and should therefore

Table 1. Summary of sample characteristics $(N=7)$

\begin{tabular}{ll}
\hline Variable & Mean $( \pm$ SD $)$ \\
\hline Height $(\mathrm{m})$ & $1.76( \pm 7.30)$ \\
Weight $(\mathrm{kg})$ & $88( \pm 14)$ \\
BMI $\left(\mathrm{kg} / \mathrm{m}^{2}\right)$ & $28.6( \pm 3.3)$ \\
Resting HR $(\mathrm{bpm})$ & $68( \pm 14)$ \\
SBP $(\mathrm{mmHg})$ & $129( \pm 12)$ \\
DBP $(\mathrm{mmHg})$ & $80( \pm 10)$
\end{tabular}

$\mathrm{SD}=$ standard deviation; $\mathrm{BMI}=$ body mass index; $\mathrm{HR}=$ heart rate; $\mathrm{SBP}=$ systolic blood pressure; DBP = diastolic blood pressure.

Table 2. Anaerobic swim test results for the $50 \mathrm{~m}$ and $2000 \mathrm{~m}$ swim protocols $(N=7)$

\begin{tabular}{|c|c|c|c|c|c|c|c|c|c|}
\hline Testing variable & $\begin{array}{l}\text { Baseline } \\
\text { mean }( \pm \mathrm{SD})\end{array}$ & $\begin{array}{l}\text { Placebo } \\
\text { mean }( \pm \text { SD })\end{array}$ & $\begin{array}{l}\text { PSE } \\
\text { mean }( \pm \text { SD })\end{array}$ & $\begin{array}{l}\text { Friedman } \\
\text { ANOVA } \\
(p \text {-value) }\end{array}$ & $\begin{array}{l}\text { Kendall co- } \\
\text { efficient of } \\
\text { concordance }\end{array}$ & $\begin{array}{l}\text { Effect } \\
\text { size }\end{array}$ & $\begin{array}{l}\text { Positive }^{*} \\
\%\end{array}$ & $\begin{array}{l}\text { Trivial* } \\
\%\end{array}$ & $\begin{array}{l}\text { Negative }^{*} \\
\%\end{array}$ \\
\hline \multicolumn{10}{|c|}{$50 \mathrm{~m}$ swim protocol } \\
\hline Time (s) & $38.8( \pm 5.6)$ & $38.7( \pm 5.5)$ & $38.6( \pm 5.4)$ & $0.92(0.6)$ & 0.7 & 0.18 & 25 & 68 & 8 \\
\hline HR (bpm) & $119( \pm 24)$ & $126( \pm 35)$ & $122( \pm 25)$ & $0.27(0.9)$ & 0.02 & 0.30 & 56 & 35 & 9 \\
\hline SBP (mmHg) & $171( \pm 27)$ & $175( \pm 27)$ & $161( \pm 27)$ & $3.2(0.2)$ & 0.2 & 0.68 & 86 & 11 & 3 \\
\hline DBP (mmHg) & $89( \pm 16)$ & $77( \pm 8)$ & $80( \pm 8)$ & $4.6(0.1)$ & 0.3 & 0.76 & 1 & 99 & 0 \\
\hline $\mathrm{RPE}$ & $7.1( \pm 2.6)$ & $7.7( \pm 2.1)$ & $7.9( \pm 1.6)$ & $1.9(0.4)$ & 0.1 & 0.16 & 1 & 99 & 0 \\
\hline \multicolumn{10}{|c|}{$2000 \mathrm{~m}$ swim protocol } \\
\hline Time (s) & $1993( \pm 550)$ & $2091( \pm 392)$ & $2079( \pm 319)$ & $1.14(0.6)$ & 0.08 & 0.06 & 52 & 8 & 41 \\
\hline HR (bpm) & $117( \pm 9)$ & $135( \pm 33)$ & $109( \pm 45)$ & $1.56(0.5)$ & 0.11 & 0.50 & 85 & 6 & 9 \\
\hline SBP (mmHg) & $166( \pm 26)$ & $170( \pm 23)$ & $157( \pm 28)$ & $1.18(0.6)$ & 0.08 & 0.47 & 76 & 17 & 7 \\
\hline $\mathrm{DBP}(\mathrm{mmHg})$ & $77( \pm 8)$ & $74( \pm 6)$ & $75( \pm 5)$ & $0.56(0.8)$ & 0.04 & 0.56 & 0 & 100 & 0 \\
\hline $\mathrm{RPE}$ & $6.9( \pm 2.5)$ & $6.9( \pm 2.40)$ & $7.1( \pm 2.1)$ & $0.95(0.95)$ & 0.007 & 0.21 & 1 & 93 & 5 \\
\hline
\end{tabular}


be avoided if suspicion is warranted. ${ }^{[14]}$ Nevertheless, the time taken for optimal effect could be extensive with the use of nutritional supplements; therefore, they are not as appealing as the faster-acting PSE. ${ }^{[15]}$

PSE is banned in-competition, due to its potentially ergogenic effect on athletes in theoretical urine concentrations $>150 \mu \mathrm{g} / \mathrm{ml}$, even though evidence of its effect in athletes is limited. ${ }^{[7,8]}$ In our study, performance was measured after a 4-day period of ingestion of $90 \mathrm{mg} / \mathrm{d}$ of PSE - an amount sufficient to cause a substantial increase in urine concentration. Furthermore, performance was tested in both sprint and long-distance events. However, the findings showed no likely enhancement in the majority of variables tested, with the exception of a probable increase in HR in the $2000 \mathrm{~m}$ swim trial (positive/trivial/negative: $85 / 6 / 9)$ with a small effect size $(d=0.1)$. The RPE subjectively measured effort level of performance, and it was ensured that the tests were conducted at high intensity ( $\mathrm{RPE} \geq 6$ ). The tests were performed using PSE and placebo in indiscriminate order initially, with these substances switched after a 4-day wash-out period.

There were slight improvements in swim time with the use of PSE in the $50 \mathrm{~m}$ and $2000 \mathrm{~m}$ events; however, the probabilty that these changes were meaningful was low ( $25 \%$ and $52 \%$, respectively). Of interest was the reduction in SBP in both tests with PSE use; an acute, but unexpected effect. This is contrary to a similar study by Chester et al., ${ }^{[4]}$ which did not show a significant change in SBP or DBP during endurance running, even with use of a higher treatment dose of PSE (240 mg). Similarly, Hunter et al. ${ }^{[16]}$ reported no influence on performance in a cycling time trial with the use of $120 \mathrm{mg}$ of PSE 2 hours prior to testing. In our study, the greatest effect size was observed in DBP during the $50 \mathrm{~m}$ sprint, but the magnitude of this effect was almost certainly trivial (99\%). The results of our study support that therapeutic PSE use does not convey quantifiable benefits during explosive anaerobic events, although the sporting discipline of our study differed from that of the aforementioned, which utilised either prolonged ${ }^{[16]}$ or endurance ${ }^{[4]}$ testing protocols. Likewise, enhancement of performance was not evident in endurance events in our study.

On the other hand, some studies have shown performance enhancement with PSE use. Hodges et al. ${ }^{[6]}$ showed an improvement in running time $(2.1 \%$; $p=0.001)$ among athletes, with no effect on HR. Apart from investigating performance in a different sport (running $\mathrm{v}$. swimming in our study), the study recruited elite athletes and employed a higher dose of PSE (180 mg v. $90 \mathrm{mg}$ in our study). The ingestion of higher than therapeutic ( $>120 \mathrm{mg}$ ) amounts of PSE may lead to increased performance, as seen in a number of other studies. ${ }^{[1,6]}$ For instance, Pritchard-Peschek et al. ${ }^{[17]}$ reported a $5.1 \%$ increase in time trial cycling performance with the ingestion of $180 \mathrm{mg}$ of PSE. The researchers attributed the improvement to stimulation of the central nervous system or alterations in metabolism, but these cannot be proven without appropriate biochemical investigations. In the same way, another study by Gill et al., ${ }^{[2]}$ which saw participants ingest 180 mg of PSE, showed an improvement in anaerobic cycling and isometric knee extension, and contrary to the present study, a significant change in HR $(p<0.001)$. Interestingly, there were no reported adverse effects. ${ }^{[2]}$ These studies support the theory that higher doses of PSE may result in enhanced performance time, which may be of relevance in swimming competitions, warranting further investigation.

Improvements could be made for future studies in this area. Firstly, a larger sample size should be used to substantiate the findings to a more comprehensive population. Secondly, future studies should include different swimming populations and should measure blood and urine concentrations of the therapeutic dosage of PSE, with the clear purpose of examining the relationship with performance. Thirdly, the swimming pool size was consistent, but the location differed. The use of one swimming pool with controlled environmental factors would ensure consistency throughout testing. The warm-up used prior to testing which varied between participants in our study, according to what they were accustomed - could be standardised for prospective studies.

\section{Conclusion}

In our study, a therapeutic dose of PSE $(90 \mathrm{mg} / \mathrm{d})$ did not show statistically significant effects on HR, SBP, swim times and RPE when ingested in therapeutic doses by masters-level swimmers. However, when the magnitude of these differences was examined, there was a relatively high probability of a decreased SBP in the $50 \mathrm{~m}$ sprint and decreased HR and SBP in the $2000 \mathrm{~m}$ swim associated with the use of PSE. Nevertheless, an ergogenic advantage does not seem to be gained from the recommended therapeutic dose of PSE $(<120 \mathrm{mg} / \mathrm{d})$ in endurance and sprint swimming performance.

\section{References}

1. Hodges ANH, Lynn BM, Bula JE, et al. Effects of pseudoephedrine on maximal cycling power and submaximal cycling efficiency. Med Sci Sports Exerc 2003;35:13161319. [http://dx.doi.org/10.1249/01.MSS.0000078925.30346.F8]

2. Gill ND, Shield A, Blazevich AJ, Zhou S, Weatherby RP. Muscular and cardiorespiratory effects of pseudoephedrine in human athletes. Br J Clin Pharmacol 2000;50:205-213. [http://dx.doi.org/10.1046/j.1365-2125.2000.00252.x]

3. Chu KS, Doherty TJ, Parise G, Milheiro JS, Tarnopolsky MA. A moderate dose of pseudoephedrine does not alter muscle contraction strength or anaerobic power. Clin J Sport Med 2002;12:387-390.

4. Chester N, Reilly T, Mottram DR. Physiological, subjective and performance effects of pseudoephedrine and phenylpropanolamine during endurance running exercise. Int J Sports Med 2003;24:3-8. [http://dx.doi.org/10.1055/s-2003-37193]

5. Salo D, Riewald SA. Complete conditioning for swimming. 1st ed. Illinois, USA: Human Kinetics, 2008.

6. Hodges K, Hancock S, Currell K, Hamilton B, Jeukendrup AE. Pseudoephedrine enhances performance in $1500 \mathrm{~m}$ runners. Med Sci Sports Exerc 2006;38:329-333. [http://dx.doi. org/10.1249/01.mss.0000183201.79330.9c]

7. World Anti-Doping Agency. The 2012 Prohibited List World Anti-Doping Code. Montreal: World Anti-Doping Agency, 2011.

8. Pokrywka A, Tszyrsznic W, Kwiatkowska DJ. Problems of the use of pseudoephedrine by athletes. Int J Sports Med 2009;30:569-572. [http://dx.doi.org/10.1055/s-0029-1202826]

9. Federation Internationale De Natation (FINA). Masters Rules. Lausanne: FINA, 2010. http://www.fina.org (accessed 9 November 2012).

10. American College of Sports Medicine (ACSM). ACSM's Guidelines for Exercise Testing and Prescription. 8th ed. Philadelphia, USA: Lippincot Williams \& Wilkins, 2010.

11. Batterham A, Hopkins W. Making inferences about magnitudes. Int J Sports Physiol Perform 2006;11(1):50-57

12. Gradidge P, Coopoo Y, Constantinou D. Attitudes and perceptions towards performance-enhancing substance use in Johannesburg boys high school sport. South African Journal of Sports Medicine 2010;22(2):32-36.

13. van der Merwe PJ, Grobbelaat E. Inadvertent doping through nutritional supplements is a reality. South African Journal of Sports Medicine 2004;16:3-7.

14. Buell JL, Franks R, Ransone J, Powers ME, Laquale KM, Carlson-Phillips A. National Athletic Trainers' Association position statement: Evaluation of dietary supplements for performance nutrition. J Athl Train 2013;48(1):124-136. [http://dx.doi. org/10.4085/1062-6050-48.1.16]

15. Gradidge P, Coopoo Y, Constantinou D. Prevalence of performance-enhancing substance use by Johannesburg male adolescents involved in competitive high school sports. Archives of Exercise in Health and Disease 2011;2(2):114-119. [http://dx.doi. org/10.5628/aehd.v2i2.102]

16. Hunter G, Derman WE, Noakes TD, Smith P, Evans A, Gabriels G. Pseudoephedrine is without ergogenic effects during prolonged exercise. J Appl Physiol 1996;81(6):2611-2617.

17. Pritchard-Peschek KR, Jenkins DG, Osborne MA, Slater GJ. Pseudoephedrine ingestion and cycling time-trial performance. Int J Sport Nutr Exerc Metab 2010;20:132-139. 